\title{
Choice of Antithrombotic Therapy for Patients with Atrial Fibrillation Undergoing Carotid Angioplasty and Stenting: A Nationwide Population-Based Study
}

Yung-Chuan Huang ( $\nabla$ richardych1111@gmail.com )

Fu Jen Catholic University

\section{Yen-Chun Huang}

Fu Jen Catholic University

\section{Yu-Chen Cheng}

Fu Jen Catholic University

Mingchih Chen

Fu Jen Catholic University

\section{Research Article}

Keywords: Atrial fibrillation, Carotid stenosis, Anticoagulation agent, Antiplatelet agent, Stroke prevention, Carotid angioplasty and stenting.

Posted Date: September 15th, 2021

DOl: https://doi.org/10.21203/rs.3.rs-880217/v1

License: (c) (i) This work is licensed under a Creative Commons Attribution 4.0 International License. Read Full License

Version of Record: A version of this preprint was published at Scientific Reports on January 26th, 2022. See the published version at https://doi.org/10.1038/s41598-022-05546-8. 


\section{Abstract}

Non-valvular atrial fibrillation (NVAF) and carotid stenosis are important risk factors for stroke. Carotid angioplasty and stent placement (CAS) is recommended for patients with symptomatic high-grade carotid stenosis. The optimal medical management for patients with NVAF after CAS remains unclear. We aimed to clarify this issue using real-world data from the Taiwanese National Health Insurance Research Database (NHIRD). A total of 2116 NVAF patients who received CAS between January 1, 2010, and December 31, 2016, from NHIRD were divided into groups based on post-procedure medication as follows: only antiplatelet agent (OAP, $n=587)$; only anticoagulation agent (OAC, $n=477)$; dual antiplatelet agents (DAP, $n=49$ ); and a combination of antiplatelet and anticoagulation agents (CAPAC, $n=304$ ). Mortality, vascular events, and major bleeding episodes were compared after matching with the Charlson comorbidity index and CHA2DS2-VASc score. The CAPAC and the OAC groups had lower mortality rates than the OAP group $(P=0.0219)$, with no statistical differences in major bleeding, ischemic stroke, or vascular events. In conclusion, OAC therapy after CAS appears suitable for NVAF patients. CAPAC therapy might be considered as initial therapy or when there is concern about vascular events.

\section{Background}

Non-valvular atrial fibrillation (NVAF) is the most common risk factor for cardiogenic stroke, with a global prevalence of approximately 1-2\% [1]. Thrombus formation in the left atrial appendage causes cerebral and systemic embolism [2]. In Asia, the stroke burden of atrial fibrillation (Af) patients in communitybased cohorts was estimated to be 13-15\% per year [3]. According to a cohort study using data from the National Health Insurance Research Database (NHIRD), Taiwanese have a high annual stroke rate exceeding 5\% when the CHA2DS2-VASc (congestive heart failure, hypertension, age 75 years or older, diabetes mellitus, previous stroke or transient ischemic attack, vascular disease, age 65-74 years, female sex) score is greater than 4 [4]. Warfarin was the standard treatment for these patients. Recently, nonvitamin $\mathrm{K}$ antagonist oral anticoagulants (NOACs) such as direct thrombin inhibitors (dabigatran) and factor Xa inhibitors have provided effective and safe protection [5-8]. However, some patients with Af and carotid stenosis have a significantly higher stroke recurrence rate than those with only Af [9]. The rupture of atherosclerotic plaques, which have a lipid core surrounded by fibrous tissue [10], may activate platelet adhesion and aggregation, blocking blood flow. Patients with severe carotid atherosclerosis have a high risk of coronary heart disease and sudden death [11]. Therefore, antiplatelet agents such as aspirin and P2Y12 inhibitors that inhibit platelet aggregation are the best choice for carotid atherosclerosis.

Carotid atherosclerosis with high-grade stenosis coexisting with NVAF is common with a $5-8 \%$ frequency in the aging population. The conditions have similar risk factors $[12,13]$. Af was recorded in more than $10 \%$ of patients who received interventions for carotid stenosis [14-16]. Concomitant Af was an independent risk factor for long-term stroke recurrence, mortality, and perioperative adverse events in these studies. For patients with severe symptomatic carotid stenosis, carotid endarterectomy or carotid angioplasty and stent placement (CAS) are the best management choices to reduce the risk of stroke [17]. 
The current consensus for treatment to prevent restenosis and vascular events after CAS is antiplatelet drugs or even dual antiplatelet drugs plus statins, with improved risk factor control.

Antiplatelet and anticoagulation agents seem equally necessary for patients with Af and severe carotid stenosis after vascular intervention. A combination of anticoagulation and dual antiplatelet agents appears to be a reasonable choice; however, there are concerns about a high bleeding risk [18]. There is recent preliminary evidence concerning a similar dilemma in the choice of medical therapy after acute coronary syndrome or percutaneous coronary intervention (PCI) in patients with Af. [19-22]. Combination therapy with one NOAC and a P2Y12 inhibitor provided adequate protection with few bleeding events in these patients. [19]. However, there are few studies focusing on antithrombotic agents after CAS, especially in NVAF patients. We aimed to conduct a preliminary evaluation of the effects of different medical therapies for this situation in real-world data.

\section{Methods}

\section{Data source}

This retrospective cohort study used claims data from the NHIRD in Taiwan. The National Health Insurance (NHI) program, which commenced on March 1, 1995, has reimbursed the health care costs of 99.9\% of Taiwan's population since 2014. The NHI program stores detailed health data, including demographic data on the insured population, consultations, diagnostic codes, and drug prescriptions. The International Classification of Diseases, Ninth Edition, Clinical Modification (ICD-9-CM) was used for recording diagnoses in NHIRD until 2016, and the Tenth Edition (ICD-10) has been used after that. Several scientific studies have used this database with high reliability. NHIRD provides a variety of personal medical information and comprehensive long-term follow-up.

\section{Ethics statement}

This research was approved by a full ethical review from Fu Jen Catholic University Institutional Review Board in Taiwan (Approval number: C104016), and all methods were carried out in accordance with relevant guidelines and regulations. The requirement for informed consent was waived by the Fu Jen Catholic University Institutional Review Board in Taiwan because due to the retrospective nature of the study and all NHIRD data had been de-identified.

\section{Study population}

We enrolled patients diagnosed with Af (ICD-9-CM code 427.31, between January 1, 2010, and December 31, 2015, or ICD-10-CM code I48, between January 1 and December 31, 2016), who received a carotid stent (operation code 33074B and 33128B) and were discharged without complications, according to medical records. Patients with a prosthetic valve (ICD-9-CM code V43.3, or ICD-10-CM codes Z95.2), venous thrombosis (ICD-9-CM code 453 or ICD-10-CM code I82), mitral valve stenosis (ICD-9-CM code 396 or ICD-10-CM codes I08) were excluded. The patients were divided into four groups: only antiplatelet agent (OAP) group, treated with aspirin or clopidogrel; only anticoagulation agent (OAC) group, treated 
with Warfarin or a NOAC, dual antiplatelet agent (DAP) group, who had aspirin plus clopidogrel and combined antiplatelet and anticoagulation agent (CAPAC) group, who were prescribed an antiplatelet agent plus an anticoagulation agent. Adherence to the secondary prevention therapy was required for at least three months in the six months following the vascular intervention (Fig. 1).

\section{Comorbidities and variable definitions}

The baseline characteristics including sex, age, Charlson comorbidity index, and CHA2DS2-VASc scores (congestive heart failure, hypertension, age 75 years or older, diabetes mellitus (DM), previous stroke or transient ischemic attack (TIA), vascular disease, age 65-74 years, female sex), and comorbidities including peripheral vascular disease (PVD), ischemic stroke or systemic embolism, hypertension (HTN), hyperlipidemia, DM, chronic liver disease, chronic kidney disease (CKD), chronic obstructive pulmonary disease (COPD), congestive heart failure (CHF) and TIA. The Charlson comorbidity index score (CCIs) is the classic index in the literature [23]. The comorbidities are weighted from 1 to 6 , and a score that reflects long-term outcomes is calculated for patients. Patients with a myocardial infarction (MI), CHF, cerebrovascular disease, connective tissue disease, PVD, dementia, COPD, DM, ulcer disease, or mild liver disease are assigned one point. Hemiplegia, moderate-to-severe renal disease, diabetes-with-end-organdamage, and any malignancy, including lymphoma and leukemia, except malignant neoplasm of the skin get two points. Three points are given for moderate or severe liver disease, while metastatic solid tumors and acquired immune deficiency syndrome are given six points.

\section{Definition of outcomes}

The primary outcome was all-cause mortality at the 1-year, 2-year, and 3-year follow-up. The secondary outcomes were vascular events, including ischemic stroke and acute MI happening within one year. We also evaluated the incidence of symptomatic hemorrhage within one year and the mortality rate over the whole follow-up period. Patients were followed up until death, vascular event, major bleeding, or the end of the study, whichever came earliest. ICD codes used to define comorbidities and clinical outcomes in our study are listed in Table 1. 
Table 1

ICD codes used to define comorbidities and clinical outcomes

\begin{tabular}{|c|c|c|c|}
\hline Disease & ICD-9 Codes & ICD-10 Codes & Definition \\
\hline Atrial fibrillation & 427.31 & 148 & $\begin{array}{l}\text { Outpatient } \\
\text { department } \\
\text { records } \geq 3\end{array}$ \\
\hline \multicolumn{4}{|c|}{ Baseline comorbidities } \\
\hline $\begin{array}{l}\text { Peripheral } \\
\text { vascular disease }\end{array}$ & $\begin{array}{l}093.0,437.3,440 . x, 441 . x, \\
443.1-443.9,47.1,557.1 \\
557.9, \text { v43.4 }\end{array}$ & $\begin{array}{l}\text { 170.x, 171.x, 173.1, 173.8, 173.9, } \\
\text { 177.1, I79.0, I79.2, K55.1, K55.8, } \\
\text { K55.9, Z95.8, Z95.9 }\end{array}$ & $\begin{array}{l}\text { Outpatient } \\
\text { department } \\
\text { records } \geq 3\end{array}$ \\
\hline $\begin{array}{l}\text { Systemic } \\
\text { embolism }\end{array}$ & 444 & 174.01-74.09 & $\begin{array}{l}\text { Outpatient } \\
\text { department } \\
\text { records } \geq 3\end{array}$ \\
\hline Hypertension & 401,402 & $|10-| 16$ & $\begin{array}{l}\text { Outpatient } \\
\text { department } \\
\text { records } \geq 3\end{array}$ \\
\hline Hyperlipidemia & 272 & E78 & $\begin{array}{l}\text { Outpatient } \\
\text { department } \\
\text { records } \geq 3\end{array}$ \\
\hline Diabetes mellitus & 250 & $\begin{array}{l}\text { E10.0, E10.1, E10.9, E11.0, } \\
\text { E11.1, E11.9 }\end{array}$ & $\begin{array}{l}\text { Outpatient } \\
\text { department } \\
\text { records } \geq 3\end{array}$ \\
\hline $\begin{array}{l}\text { Chronic liver } \\
\text { disease }\end{array}$ & $570,571,572$ & $\begin{array}{l}\text { B150, B160, B162, B190, } \\
\text { K704, K72, K766, I85 }\end{array}$ & $\begin{array}{l}\text { Outpatient } \\
\text { department } \\
\text { records } \geq 3\end{array}$ \\
\hline Ischemic stroke & $433,434,436$ & 163,164 & $\begin{array}{l}\text { Outpatient } \\
\text { department } \\
\text { records } \geq 3\end{array}$ \\
\hline $\begin{array}{l}\text { Chronic kidney } \\
\text { disease }\end{array}$ & $580-589$ & $\begin{array}{l}\text { I12, I13, N00, N01, N02, N03, N04, } \\
\text { N05, } \\
\text { N07, N11, N14, N17, N18, N19, } \\
\text { Q61 }\end{array}$ & $\begin{array}{l}\text { Outpatient } \\
\text { department } \\
\text { records } \geq 3\end{array}$ \\
\hline
\end{tabular}




\begin{tabular}{|c|c|c|c|}
\hline Disease & ICD-9 Codes & ICD-10 Codes & Definition \\
\hline $\begin{array}{l}\text { Chronic lung } \\
\text { disease }\end{array}$ & $\begin{array}{l}\text { 490, 491.0, 491.1, } \\
491.20-491.22, \\
491.8,491.9,492.0,492.8, \\
493.00-493.02493 .10- \\
493.12, \\
493.20-493.22,493.81, \\
493.82,493.90-493.92, \\
494.0, \\
494.1,495.8,495.9,496, \\
500,502,503,504,505, \\
\text { A323, A325 }\end{array}$ & J44 & $\begin{array}{l}\text { Outpatient } \\
\text { department } \\
\text { records } \geq 3\end{array}$ \\
\hline $\begin{array}{l}\text { Congestive heart } \\
\text { failure }\end{array}$ & 428 & $\begin{array}{l}\text { I11.0, I13.0, I13.2, I42.0, } \\
150,150.1,150.9\end{array}$ & $\begin{array}{l}\text { Outpatient } \\
\text { department } \\
\text { records } \geq 3\end{array}$ \\
\hline $\begin{array}{l}\text { Transient } \\
\text { ischemic attack }\end{array}$ & 435 & G45 & $\begin{array}{l}\text { Outpatient } \\
\text { department } \\
\text { records } \geq 3\end{array}$ \\
\hline $\begin{array}{l}\text { Percutaneous } \\
\text { coronary } \\
\text { intervention }\end{array}$ & \multicolumn{2}{|c|}{$\begin{array}{l}\text { procedure codes of the Taiwan NHI: 33076A, 33076B, 33077A, } \\
33077 \mathrm{~B}, 33078 \mathrm{~A}, 33078 \mathrm{~B}\end{array}$} & Discharge \\
\hline Malignancy & $140.0-208.9$ & $\mathrm{C}$ & $\begin{array}{l}\text { Outpatient } \\
\text { department } \\
\text { records } \geq 2\end{array}$ \\
\hline Clinical outcomes & & & \\
\hline $\begin{array}{l}\text { New ischemic } \\
\text { stroke (ischemic } \\
\text { stroke, transient } \\
\text { ischemic attack) }\end{array}$ & $\begin{array}{l}433,434,436 \\
435\end{array}$ & $\begin{array}{l}163,164 \\
G 45\end{array}$ & Discharge \\
\hline $\begin{array}{l}\text { Myocardial } \\
\text { infarction }\end{array}$ & $410,411,412$ & $121-125$ & Discharge \\
\hline
\end{tabular}




\begin{tabular}{|c|c|c|c|}
\hline Disease & ICD-9 Codes & ICD-10 Codes & Definition \\
\hline \multirow{12}{*}{$\begin{array}{l}\text { Bleeding } \\
\text { (intracranial } \\
\text { hemorrhage, } \\
\text { gastrointestinal } \\
\text { and other critical } \\
\text { site bleeding }\end{array}$} & $430,431,432,852,853$ & $160,161,162$ & Discharge \\
\hline & $456.0,456.2,455.2,455.5$, & \multirow{11}{*}{$\begin{array}{l}\text { K250, K260, K270, K280, K290 } \\
\text { D62, J942, H113, H356, H431, } \\
\text { N02, N95, R04, R31, R58 }\end{array}$} & \\
\hline & 5307530825210 & & \\
\hline & 531.6, & & \\
\hline & $\begin{array}{l}532.0-532.6,533.0- \\
533.6\end{array}$ & & \\
\hline & $\begin{array}{l}534.0-534.6,535.0- \\
535.6537 .83,\end{array}$ & & \\
\hline & $\begin{array}{l}562.02,562.03,562.12 \\
562.13\end{array}$ & & \\
\hline & $\begin{array}{l}\text { 568.81, 569.3, 569.85, } \\
\text { 578.0, }\end{array}$ & & \\
\hline & $578.1,578.9$ & & \\
\hline & $\begin{array}{l}423,0,459.0,568.81 \\
593.81\end{array}$ & & \\
\hline & $599.7,623.8,626.32$ & & \\
\hline & $\begin{array}{l}626.6,719.1,784.7,784.8, \\
786.3\end{array}$ & & \\
\hline
\end{tabular}

\section{Statistical analysis}

This research used the Chi-square test for categorical variables (shown as numbers and percentages; $\mathrm{N}$ $\%$ ) and ANOVA for continuous variables (shown as mean and standardized differences; mean \pm SD) to analyze three different medication groups according to baseline demographics. We used 1:1 ratio propensity score matching to reduce the effect of confounding factors. The variables included age, sex, CHA2DS2-VASc scores, and history of disease (Table 1). The Kaplan-Meier method was used to evaluate time-to-death outcomes from the index date and the Cox proportional hazard regression model to estimate the hazard ratios (HR), 95\% confidence interval (Cls) for mortality rates up to 3 years. We used SAS 9.4 software (SAS Institute, Cary, NC, USA). In the two-tailed tests, $P<0.05$ indicated statistical significance.

\section{Results}

After exclusions, we enrolled 2116 consecutive NVAF patients who received successful CAS with regular follow-up between January 1, 2010, and December 31, 2016. A total of 587, 477, 49, and 304 patients took OAP, OAC, DAP, and CAPAC with persistent drug adherence. The data showed a diversity of drug choice, which suggested difficulty in drug selection. Moreover, around one-third of all patients $(n=733)$ had inconsistent treatment records. The leading causes of mortality were vascular events, accounting for 
more than one-third of all deaths. Patients who took OAP or OAC were older and had relatively higher CHA2DS2-VASc scores than patients who took CAPAC (Table 2). Moreover, there was a higher proportion of comorbidities like DM, CKD, and COPD in the OAP group patients. The final analysis was based on cross-comparison after matching between groups except the DAP group because there were not enough patients who took dual antiplatelet agents consistently after CAS to match with other groups. The CHA2DS2-VASc score is an excellent evaluation tool for the risk of stroke in patients with NVAF [24]. It is also effective in predicting the clinical outcomes of patients undergoing CAS [25]. We matched different therapy groups mainly according to age, sex, and CHA2DS2-VASc scores to compare primary outcomes. The OAP group showed a higher mortality rate in the acute stage and at the end of follow-up compared with the CAPAC and OAC groups (Table 3 and Fig. 2). Administration of an anticoagulation agent seemed to be an essential protective factor influencing 3-year mortality with a hazard ratio of 0.624 (Table 4). There was no significant difference between these three groups in major bleeding, ischemic stroke, or vascular events in the acute stage. However, there was a trend toward fewer vascular events, especially ischemic stroke, but more bleedings, observed in the CAPAC group. 
Table 2

Baseline characteristics of non-valvular atrial fibrillation patients who had carotid angioplasty and stent placement (CAS), according to treatment type

\begin{tabular}{|c|c|c|c|c|c|c|c|c|}
\hline \multicolumn{2}{|c|}{ Variables } & \multicolumn{7}{|c|}{ Before matching } \\
\hline & & \multicolumn{2}{|c|}{$\begin{array}{l}\text { only } \\
\text { antiplatelet } \\
\text { agent ( } \mathrm{N}= \\
587)\end{array}$} & \multicolumn{2}{|c|}{$\begin{array}{l}\text { Only } \\
\text { anticoagulation } \\
\text { agent }(\mathrm{N}=477)\end{array}$} & \multicolumn{2}{|c|}{$\begin{array}{l}\text { A combination of antiplatelet } \\
\text { and anticoagulation agents }(\mathrm{N} \\
=304)\end{array}$} & \multirow[t]{2}{*}{$\begin{array}{l}p- \\
\text { value }\end{array}$} \\
\hline & & $\mathbf{n}$ & $\%$ & $\mathbf{n}$ & $\%$ & $\mathbf{N}$ & $\%$ & \\
\hline \multirow[t]{2}{*}{ Gender } & Female & 224 & 38.16 & 232 & 48.64 & 119 & 39.14 & 0.0014 \\
\hline & Male & 363 & 61.84 & 245 & 51.36 & 185 & 60.86 & \\
\hline \multicolumn{2}{|c|}{$\begin{array}{l}\text { Age, mean (SD) } \\
\text { year }\end{array}$} & \multicolumn{2}{|c|}{73.79 (9.29) } & \multicolumn{2}{|c|}{ 73.13(10.22) } & \multicolumn{2}{|c|}{$71.63(11.03)$} & 0.008 \\
\hline \multicolumn{2}{|c|}{$\begin{array}{l}\text { Total cost } \\
\text { (NT\$1000) }\end{array}$} & \multicolumn{2}{|c|}{$\begin{array}{l}172,839 \\
(148,860)\end{array}$} & \multicolumn{2}{|c|}{$\begin{array}{l}188,872 \\
(202.553)\end{array}$} & \multicolumn{2}{|c|}{$190,859(165,104)$} & 0.2873 \\
\hline \multicolumn{2}{|c|}{$\begin{array}{l}\text { CHA2DS2-VASc } \\
\text { score }\end{array}$} & \multicolumn{2}{|c|}{$4.261(1.613)$} & \multicolumn{2}{|c|}{$4.092(1.690)$} & \multicolumn{2}{|c|}{$3.783(1.710)$} & 0.0002 \\
\hline \multicolumn{9}{|c|}{ Baseline (previous history) } \\
\hline \multicolumn{2}{|l|}{ PVD } & 267 & 45.49 & 181 & 37.95 & 112 & 36.84 & 0.0117 \\
\hline \multicolumn{2}{|c|}{$\begin{array}{l}\text { Systemic } \\
\text { embolism }\end{array}$} & 114 & 19.42 & 113 & 23.69 & 69 & 22.7 & 0.2136 \\
\hline \multicolumn{2}{|c|}{ Hypertension } & 209 & 35.6 & 157 & 32.91 & 97 & 31.91 & 0.4710 \\
\hline \multicolumn{2}{|c|}{ Hyperlipidemia } & 365 & 62.18 & 257 & 53.88 & 156 & 51.32 & 0.0021 \\
\hline \multicolumn{2}{|c|}{ Diabetes mellitus } & 346 & 58.94 & 211 & 44.23 & 124 & 40.79 & $<.001$ \\
\hline \multicolumn{2}{|c|}{ Liver cirrhosis } & 23 & 3.92 & 16 & 3.35 & 10 & 3.29 & 0.8441 \\
\hline \multicolumn{2}{|l|}{ Stroke } & 313 & 53.32 & 270 & 56.60 & 161 & 52.96 & 0.4813 \\
\hline \multicolumn{2}{|l|}{ CKD } & 217 & 36.97 & 113 & 23.69 & 64 & 21.05 & $\dot{0} 001$ \\
\hline \multicolumn{2}{|l|}{ COPD } & 309 & 52.64 & 237 & 49.69 & 136 & 44.74 & $<.001$ \\
\hline \multicolumn{2}{|l|}{$\mathrm{CHF}$} & 381 & 64.91 & 296 & 62.05 & 190 & 62.5 & 0.5911 \\
\hline \multicolumn{9}{|c|}{$\begin{array}{l}\text { NOAC: Non-vitamin K antagonist oral anticoagulant; NTS: New Taiwan dollar; SD: standard deviation; } \\
\text { CHA2DS2-VASc: congestive heart failure, hypertension, age } 75 \text { years or older, diabetes mellitus, } \\
\text { previous stroke/transient ischemic attack, vascular disease, age } 65 \text { to } 74 \text { years, female; PVD: } \\
\text { peripheral vascular disease; CKD: chronic kidney disease; COPD: chronic obstructive pulmonary } \\
\text { disease; CHF: congestive heart failure; TIA: transient ischemic attack; PCI: percutaneous coronary } \\
\text { intervention }\end{array}$} \\
\hline
\end{tabular}




\begin{tabular}{|c|c|c|c|c|c|c|c|}
\hline \multirow[t]{3}{*}{ Variables } & \multicolumn{7}{|c|}{ Before matching } \\
\hline & \multicolumn{2}{|c|}{$\begin{array}{l}\text { only } \\
\text { antiplatelet } \\
\text { agent ( } \mathrm{N}= \\
587)\end{array}$} & \multicolumn{2}{|c|}{$\begin{array}{l}\text { Only } \\
\text { anticoagulation } \\
\text { agent }(\mathrm{N}=477)\end{array}$} & \multicolumn{2}{|c|}{$\begin{array}{l}\text { A combination of antiplatelet } \\
\text { and anticoagulation agents }(\mathrm{N} \\
=304)\end{array}$} & \multirow[t]{2}{*}{$\begin{array}{l}p- \\
\text { value }\end{array}$} \\
\hline & $\mathrm{n}$ & $\%$ & $\mathrm{n}$ & $\%$ & $\mathbf{N}$ & $\%$ & \\
\hline TIA & 313 & 53.32 & 272 & 57.02 & 161 & 52.96 & 0.3979 \\
\hline $\mathrm{PCl}$ & 42 & 7.16 & 13 & 3.19 & 12 & 3.95 & 0.0027 \\
\hline \multicolumn{8}{|c|}{$\begin{array}{l}\text { NOAC: Non-vitamin K antagonist oral anticoagulant; NTS: New Taiwan dollar; SD: standard deviation; } \\
\text { CHA2DS2-VASc: congestive heart failure, hypertension, age } 75 \text { years or older, diabetes mellitus, } \\
\text { previous stroke/transient ischemic attack, vascular disease, age } 65 \text { to } 74 \text { years, female; PVD: } \\
\text { peripheral vascular disease; CKD: chronic kidney disease; COPD: chronic obstructive pulmonary } \\
\text { disease; CHF: congestive heart failure; TIA: transient ischemic attack; PCI: percutaneous coronary } \\
\text { intervention }\end{array}$} \\
\hline
\end{tabular}


Table 3

Clinical outcomes of different therapeutic groups after matching by age, sex, CHA2DS2-VASc score and baseline comorbidities

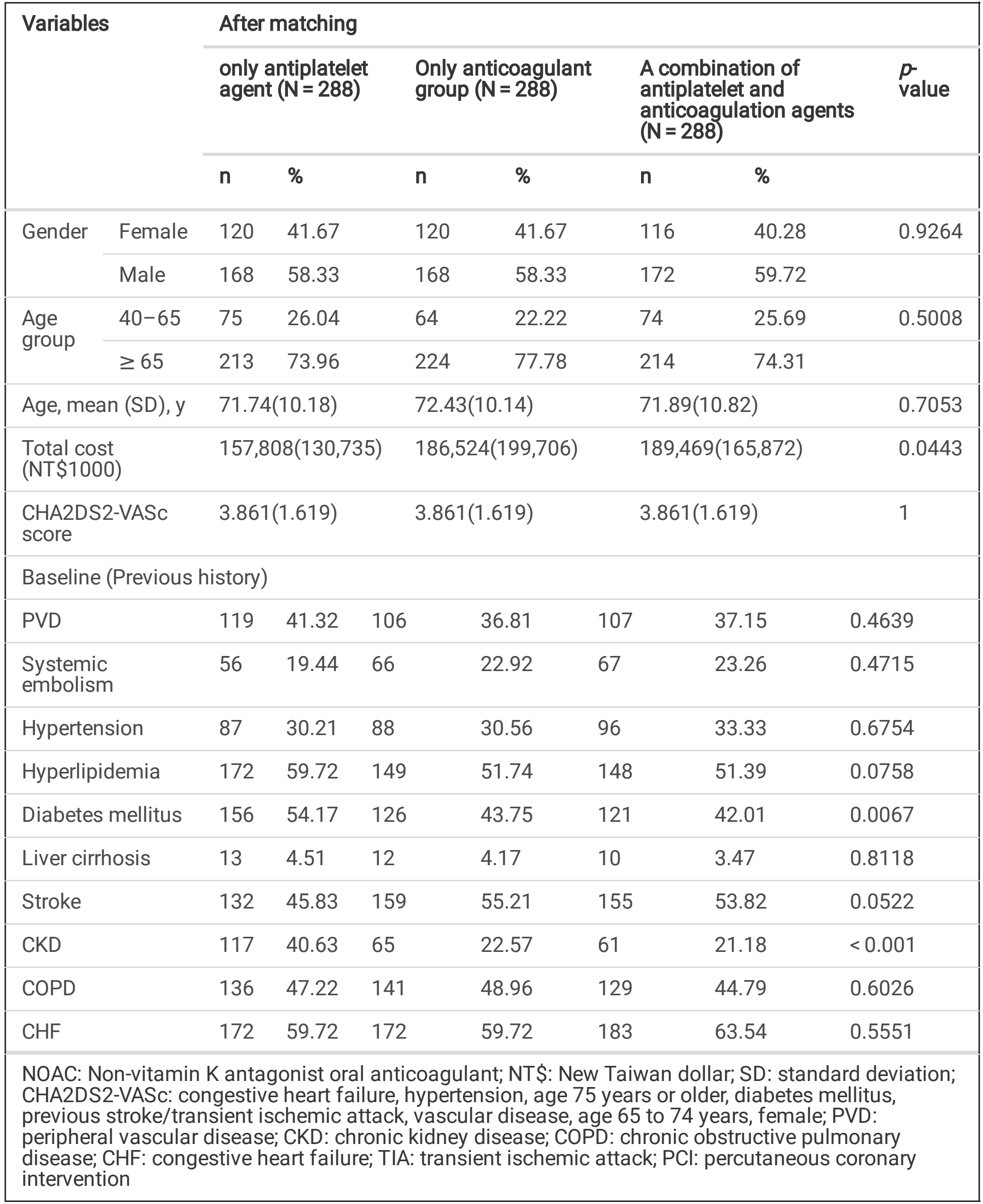




\begin{tabular}{|c|c|c|c|c|c|c|c|}
\hline \multirow[t]{3}{*}{ Variables } & \multicolumn{7}{|c|}{ After matching } \\
\hline & \multicolumn{3}{|c|}{$\begin{array}{l}\text { only antiplatelet } \\
\text { agent }(\mathrm{N}=288)\end{array}$} & \multicolumn{2}{|c|}{$\begin{array}{l}\text { Only anticoagulant } \\
\text { group }(\mathrm{N}=288)\end{array}$} & $\begin{array}{l}\text { A combination of } \\
\text { antiplatelet and } \\
\text { anticoagulation agents } \\
(\mathrm{N}=288)\end{array}$ & $p$ value \\
\hline & $\mathbf{n}$ & $\%$ & $r$ & $\%$ & I & $\%$ & \\
\hline TIA & 132 & 45.83 & 160 & 55.56 & 155 & 53.82 & 0.045 \\
\hline $\mathrm{PCl}$ & 23 & 7.99 & 11 & 3.82 & 11 & 3.82 & 0.034 \\
\hline \multicolumn{8}{|l|}{ Outcome } \\
\hline $\begin{array}{l}\text { Mortality }(0-1 Y) \\
(n)\end{array}$ & 36 & 12.50 & 31 & 10.76 & 31 & 10.76 & 0.7500 \\
\hline $\begin{array}{l}\text { Mortality }(0-2 Y) \\
(n)\end{array}$ & 77 & 26.74 & 66 & 22.92 & 61 & 21.18 & $<0.001$ \\
\hline $\begin{array}{l}\text { Mortality }(0-3 Y) \\
(n)\end{array}$ & 103 & 35.76 & 92 & 31.94 & 84 & 29.17 & 0.2357 \\
\hline $\begin{array}{l}\text { Mortality during } \\
\text { whole follow-up }\end{array}$ & 187 & 64.93 & 155 & 53.82 & 176 & 61.11 & 0.0219 \\
\hline $\begin{array}{l}\text { New ischemic } \\
\text { stroke }\end{array}$ & 18 & 6.25 & 19 & 6.6 & 11 & 3.82 & 0.2844 \\
\hline $\begin{array}{l}\text { Myocardial } \\
\text { infarction }\end{array}$ & 11 & 3.82 & 12 & 4.17 & 12 & 4.17 & 0.9707 \\
\hline Vascular events & 29 & 10.07 & 31 & 10.76 & 23 & 7.99 & 0.5 \\
\hline Bleeding & 103 & 35.7 & 117 & 40.6 & 129 & 44.8 & 0.087 \\
\hline \multicolumn{8}{|c|}{$\begin{array}{l}\text { NOAC: Non-vitamin K antagonist oral anticoagulant; NTS: New Taiwan dollar; SD: standard deviation; } \\
\text { CHA2DS2-VASc: congestive heart failure, hypertension, age } 75 \text { years or older, diabetes mellitus, } \\
\text { previous stroke/transient ischemic attack, vascular disease, age } 65 \text { to } 74 \text { years, female; PVD: } \\
\text { peripheral vascular disease; CKD: chronic kidney disease; COPD: chronic obstructive pulmonary } \\
\text { disease; CHF: congestive heart failure; TIA: transient ischemic attack; PCI: percutaneous coronary } \\
\text { intervention }\end{array}$} \\
\hline
\end{tabular}


Table 4

Cox proportional model to estimate the hazard ratios of 3-year mortality

\begin{tabular}{|c|c|c|c|}
\hline Variables & & $\begin{array}{l}\text { Adjusted HR (95\% } \\
\mathrm{Cl})\end{array}$ & $\begin{array}{l}p- \\
\text { value }\end{array}$ \\
\hline \multicolumn{4}{|l|}{ Gender } \\
\hline Female & Ref & 1 & \\
\hline Male & & $0.893(0.683-1.166)$ & 0.4042 \\
\hline \multicolumn{4}{|l|}{ Age } \\
\hline $40-65$ & Ref & 1 & \\
\hline$\geq 65$ & & $1.246(0.875-1.773)$ & 0.2223 \\
\hline \multicolumn{4}{|l|}{ CHA2DS2-VASc score } \\
\hline$<4$ & Ref & 1 & \\
\hline$\geq 4$ & & $1.217(0.841-1.761)$ & 0.2982 \\
\hline \multicolumn{4}{|l|}{ Group } \\
\hline Only antiplatelet agent & Ref & 1 & \\
\hline Only anticoagulation agent & & $0.624(0.459-0.849)$ & 0.0096 \\
\hline $\begin{array}{l}\text { A combination of antiplatelet and anticoagulation } \\
\text { agents }\end{array}$ & & $0.846(0.622-1.151)$ & 0.2869 \\
\hline COPD & $\begin{array}{l}\text { (ref: } \\
\text { No) }\end{array}$ & $1.213(0.938-1.568)$ & 0.1402 \\
\hline CKD & & $0.887(0.687-1.146)$ & 0.358 \\
\hline $\mathrm{CHF}$ & & $1.122(0.823-1.529)$ & 0.4675 \\
\hline Diabetes & & $1.295(0.986-1.702)$ & 0.0633 \\
\hline Hypertension & & $0.748(0.57-0.983)$ & 0.0376 \\
\hline Hyperlipidemia & & $0.904(0.697-1.172)$ & 0.4443 \\
\hline Stroke & & $1.143(0.869-1.504)$ & 0.3395 \\
\hline Ischemic heart & & $0.864(0.544-1.374)$ & 0.5379 \\
\hline
\end{tabular}

\section{Discussion}


In-stent restenosis and cardioembolic stroke should be prevented in patients with NVAF who receive CAS for symptomatic high-grade carotid stenosis. Even after CAS, patients with a history of severe carotid atherosclerosis have a high risk of composite vascular events such as $\mathrm{Ml}$ and vascular death. The combination of antiplatelet and anticoagulation agents might be a reasonable choice for these patients after intervention for the coexisting risks of atherosclerotic and cardioembolic events in these patients. There has been much discussion about combining antiplatelet and anticoagulation agents for Af and thrombotic events in cardiovascular disease [20-22, 26]. According to recent cohort studies, for patients with Af after acute coronary syndrome or $\mathrm{PCl}$, triple therapy with dual antiplatelet and one anticoagulation agent should be used briefly, with caution. Then changing to treatment with one NOAC and P2Y12 inhibitor is recommended [19]. Cardiovascular and cerebrovascular diseases have a similar etiology and pathophysiology. However, a recent study investigating combination therapy for stable peripheral or carotid artery disease showed the benefit of reducing major adverse limb events at the cost of increasing major bleeding [27]. There are no guidelines or extensive cohort studies providing evidence on whether to follow the same track in managing patients with coexisting Af and carotid artery stenosis.

To our knowledge, this is the first study to focus on antiplatelet and anticoagulation agents after CAS in patients with underlying NVAF. According to our NHIRD 2008-2017 data, Taiwanese patients with coexisting Af and symptomatic carotid stenosis had a high mortality rate after CAS, and less than half were alive after five years. Vascular events were the primary cause of death even with intensive regular medical prevention. The lack of a standard treatment is shown by the divergent drug selection in our data.

Several periprocedural complications, such as hyperperfusion syndrome, gastrointestinal hemorrhage, MI, systemic embolism, stroke, and TIA, occurred frequently within 30 days of CAS with an incidence of around $4 \%$ [28]. These events during admission for CAS may be difficult to determine from our data and might have influenced drug choice. Our study focused on the drug choice for patients with NVAF one month after CAS. In comparing primary outcomes, a combination of antiplatelet and anticoagulation agents or only anticoagulation agents in the acute stage seemed more helpful for these patients than only an antiplatelet agent. A lower incidence of vascular events, especially ischemic stroke, was observed in the combination group. There was an increased risk of bleeding, which was not statistically significant. A strategy focusing on anticoagulation effects plays a vital role in this situation. Compared to the previous study focusing on antithrombotic agents after acute coronary syndrome or $\mathrm{PCl}$ in $\mathrm{Af}$, our analysis showed fewer vascular events but more bleeding [22]. As we previously mentioned, most in-stent restenosis and vascular events happened within the first month in patients without NVAF receiving CAS who would not be included in our study. Further, our study was based on observational data according to the admission ICD codes, therefore, some minor strokes or vascular events without admission might have been missed. However, overall mortality rates may reflect the sum of these physical problems. Treatments containing anticoagulation agents must be implemented in clinical practice in patients with NVAF receiving CAS. A combination of a single antiplatelet and an anticoagulation agent might be indicated when there are concerns about possible vascular events. It might be beneficial to initially prescribe combination therapy during the high-risk period. Kouhei also suggested administering one NOAC plus a 
single antiplatelet agent in a published case series. Clopidogrel may be a good choice of antiplatelet agent according to studies of $\mathrm{PCl}[22,29]$. Our study did not separate patients taking aspirin and warfarin or clopidogrel and NOACs, which might be the reason more bleeding was recorded.

Current European guidelines recommend lifelong OAC with no antiplatelet agent beyond one year after coronary stenting in patients with NVAF [30]. Anticoagulation monotherapy was superior to aspirin or clopidogrel alone in preventing ischemic events. An anticoagulant plus a single antiplatelet agent did not improve protection but increased the risk of bleeding after the acute stage of Ml. Our study showed that a single anticoagulation agent provided better prevention than a single antiplatelet agent and had a lower bleeding risk than combination therapy. Prevention of vascular events and bleeding risk resulted in low mortality during follow-up, and most hazard ratio reduction was recorded in the only anticoagulation therapy group. One anticoagulant seemed to be enough for most cases with NVAf after CAS.

Based on the experience of previous clinical trials, the use of dual antiplatelet drugs for a short period is the consensus for medical management after CAS [31]. But these trials were non-blinded and had small numbers [32]. The appropriate duration of dual antiplatelet therapy after CAS is unknown. In line with the existing guidelines for the period covered by our study, dual antiplatelet agents were prescribed for one month after intervention. Therefore, in our study, most physicians stopped dual antiplatelet agents after 1 month, and there were only 49 cases that were prescribed dual antiplatelet agents consistently in the halfyear following carotid stenting. According to experience from studies focusing on acute coronary syndrome or $\mathrm{PCl}$ in Af, triple therapy with dual antiplatelet agents and one anticoagulation agent is unnecessary. It increases bleeding risk one month after the intervention for most cases. These two kinds of medicine selection were not considered in our study.

This study has several limitations. It is only an observational study based on NHIRD data. The identification of comorbidities and complications is based on diagnostic codes listed in the database; some coding errors may have occurred. A diagnostic bias concerning the endpoints can be ruled out due to specific operation codes and ICD codes for significant events being checked carefully during admission according to health insurance requirements. The disease severity and any underlying diseases may affect the physician's drug choice. To reduce the observational study's inherent bias, we matched patients according to age, CHA2DS2-VASc score, and comorbidities before analysis where possible. There are no clear post-CAS drug treatment guidelines, especially for patients with NVAF. Prescribing may vary and, at times, were changed due to the clinical physicians' choice. We decided to group the patients according to drug use information obtained from the outpatient pharmacy prescription database. Only patients who took one kind of medicine continuously for at least half a year were enrolled. However, drug compliance and the influence of lifestyle factors such as smoking, overeating, and poor sleep quality could not be accounted for in this study.

In summary, this study may offer preliminary evidence for clinical practice and insights for further investigation. One anticoagulation agent had a lower mortality rate and relatively low bleeding risk. The Cox proportional model estimate of the HR showed that drug choice of one anticoagulation agent might 
be the critical factor influencing mortality at 3-year follow-up. One anticoagulation agent may be standard medical therapy after CAS in patients with NVAF. Combination therapy with one antiplatelet and one anticoagulation agent may be considered as initial therapy when there are concerns about possible vascular events. NOACs might be a better choice than warfarin, according to some studies in Asian populations [33-35]. Clopidogrel may be a good choice of antiplatelet agent.

Prompt intervention followed by intensive medical control with anticoagulation therapy is the most reasonable choice for patients with NVAF and high-grade carotid stenosis. Further large-scale randomized trials are needed to confirm this.

\section{Declarations}

Acknowledgements: The authors would like to thank the editors and the reviewers for their valuable comment. The auther sincerely appreciate NHIRD, which was provided by the Ministry of Health and Welfare.

Authors' contributions: Conception and design: Yung-Chuan Huang and Y.-C. Cheng; data collection, methods, analysis and interpretation: Yen-Chun Huang and M. Chen; drafting of the manuscript: YungChuan Huang and Yen-Chun Huang; overall responsibility: Yung-Chuan Huang and M. Chen.

Funding: This study did not receive external funding.

Conflicts of interest: There are no conflicts of interest to declare

Ethics approval: The requirement for informed consent was waived by the Fu Jen Catholic University Institutional Review Board (C108121) in Taiwan because due to the retrospective nature of the study and all NHIRD data had been de-identified.

\section{References}

1. Go, A. S. et al. Prevalence of diagnosed atrial fibrillation in adults: national implications for rhythm management and stroke prevention: the AnTicoagulation and Risk Factors in Atrial Fibrillation (ATRIA) Study. JAMA, 285, 2370-2375 (2001).

2. January, C. T. et al. 2014 AHA/ACC/HRS guideline for the management of patients with atrial fibrillation: executive summary: a report of the American College of Cardiology/American Heart Association Task Force on practice guidelines and the Heart Rhythm Society., 130, 2071-2104 (2014).

3. Lip, G. Y. H., Brechin, C. M. \& Lane, D. A. The global burden of atrial fibrillation and stroke: a systematic review of the epidemiology of atrial fibrillation in regions outside North America and Europe., 142, 1489-1498 (2012).

4. Chao, T. F. et al. Using the CHA2DS2-VASc score for refining stroke risk stratification in 'low-risk' Asian patients with atrial fibrillation. J. Am. Coll. Cardiol, 64, 1658-1665 (2014). 
5. Connolly, S. J. et al. Dabigatran versus warfarin in patients with atrial fibrillation. N. Engl. J. Med, 361, 1139-1151 (2009).

6. Patel, M. R. et al. Rivaroxaban versus warfarin in nonvalvular atrial fibrillation. N. Engl. J. Med, 365, 883-891 (2011).

7. Granger, C. B. et al. Apixaban versus warfarin in patients with atrial fibrillation. N. Engl. J. Med, 365, 981-992 (2011).

8. Giugliano, R. P. et al. Edoxaban versus warfarin in patients with atrial fibrillation. N. Engl. J. Med, 369, 2093-2104 (2013).

9. Lehtola, H. et al. Stroke recurrence in patients with atrial fibrillation: concomitant carotid artery stenosis doubles the risk. Eur. J. Neurol, 24, 719-725 (2017).

10. Castelli, W. P. Cholesterol and lipids in the risk of coronary artery disease-the Framingham Heart Study. Can. J. Cardiol. 4 Suppl A, 5A-10A(1988).

11. Goessens, B. M. B., Visseren, F. L. J., Kappelle, L. J., Algra, A. \& van der Graaf, Y. Asymptomatic carotid artery stenosis and the risk of new vascular events in patients with manifest arterial disease: the SMART study., 38, 1470-1475 (2007).

12. Akanksha, W. G. et al. Clinical Features, Risk Factors, and Short-term Outcome of Ischemic Stroke, in Patients with Atrial Fibrillation: Data from a Population-based Study. Ann. Indian Acad. Neurol, 20, 289-293 (2017).

13. Yang, P. S. et al. Non-cardioembolic risk factors in atrial fibrillation-associated ischemic stroke. PLoS One, 13, e0201062 (2018).

14. Watanabe, M. et al. The effect of atrial fibrillation on outcomes in patients undergoing carotid endarterectomy or stent placement in general practice. J. Vasc. Surg, 61, 927-932 (2015).

15. Mazzaccaro, D., Modafferi, A., Malacrida, G. \& Nano, G. Assessment of long-term survival and stroke after carotid endarterectomy and carotid stenting in patients older than 80 years. J. Vasc. Surg, 70, 522-529 (2019).

16. Qureshi, A. I., Chaudhry, S. A., Qureshi, M. H. \& Suri, M. F. K. Rates and predictors of 5-year survival in a national cohort of asymptomatic elderly patients undergoing carotid revascularization. Neurosurgery, 76, 34-40 discussion 40-1 (2015).

17. Brott, T. G. et al. 2011

ASA/ACCF/AHA/AANN/AANS/ACR/ASNR/CNS/SAIP/SCAI/SIR/SNIS/SVM/SVS guideline on the management of patients with extracranial carotid and vertebral artery disease: executive summary. A report of the, American Association of Neuroscience Nurses, American Association of Neurological Surgeons, American College of Radiology, American Society of Neuroradiology, Congress of Neurological Surgeons, Society of Atherosclerosis Imaging and Prevention, Society for Cardiovascular Angiography and Interventions, Society of Interventional Radiology, Society of Neurolnterventional Surgery, Society for Vascular Medicine, and Society for Vascular Surgery. Circulation 124, 489-532 (2011). 
18. Vandiver, J. W. \& Diane Beavers, K. Combining oral anticoagulation and antiplatelet therapies: appropriate patient selection. J. Thromb. Thrombolysis, 45, 423-431 (2018).

19. Collet, J. P. et al. 2020 ESC Guidelines for the management of acute coronary syndromes in patients presenting without persistent ST-segment elevation. Eur. Heart J, 42, 1289-1367 (2021).

20. Lopes, R. D. et al. Safety and Efficacy of Antithrombotic Strategies in Patients With Atrial Fibrillation Undergoing Percutaneous Coronary Intervention: A Network Meta-analysis of Randomized Controlled Trials. JAMA Cardiol, 4, 747-755 (2019).

21. Cannon, C. P. et al. Dual Antithrombotic Therapy with Dabigatran after PCl in Atrial Fibrillation. N. Engl. J. Med, 377, 1513-1524 (2017).

22. Lopes, R. D. et al. Antithrombotic Therapy after Acute Coronary Syndrome or PCl in Atrial Fibrillation. N. Engl. J. Med, 380, 1509-1524 (2019).

23. Yang, H., Chen, Y. H., Hsieh, T. F., Chuang, S. Y. \& Wu, M. J. Prediction of Mortality in Incident Hemodialysis Patients: A Validation and Comparison of CHADS2, CHA2DS2, and CCI Scores. PLoS One, 11, e0154627 (2016).

24. Friberg, L., Rosenqvist, M. \& Lip, G. Y. H. Evaluation of risk stratification schemes for ischaemic stroke and bleeding in 182678 patients with atrial fibrillation: the Swedish Atrial Fibrillation cohort study. Eur. Heart J, 33, 1500-1510 (2012).

25. Cerşit, S. et al. Usefulness of CHA2DS2-VASc Score to predict clinical outcomes of patients undergoing carotid artery stenting. Int. J. Cardiovasc. Imaging, 37, 783-789 (2021).

26. Saito, Y. \& Kobayashi, Y. Triple, dual, and single antithrombotic therapy for patients with atrial fibrillation undergoing percutaneous coronary intervention. Cardiovasc. Interv. Ther, 35, 44-51 (2020).

27. Anand, S. S. et al. Rivaroxaban with or without aspirin in patients with stable peripheral or carotid artery disease: an international, randomised, double-blind, placebo-controlled trial., 391, 219-229 (2018).

28. Sakai, N. et al. Prospective registry of carotid artery stenting in Japan-investigation on device and antiplatelet for carotid artery stenting. J. Stroke Cerebrovasc. Dis, 23, 1374-1384 (2014).

29. Dewilde, W. J. M. et al. Use of clopidogrel with or without aspirin in patients taking oral anticoagulant therapy and undergoing percutaneous coronary intervention: an open-label, randomised, controlled trial., 381, 1107-1115 (2013).

30. Lip, G. Y. H. et al. 2018 joint European consensus document on the management of antithrombotic therapy in atrial fibrillation patients presenting with acute coronary syndrome and/or undergoing percutaneous cardiovascular interventions: a joint consensus document of the European heart rhythm association (EHRA), European Society of cardiology Working group on thrombosis, European association of percutaneous cardiovascular interventions (EAPCI), and European association of acute cardiac care (ACCA) endorsed by the heart rhythm Society (HRS), Asia-Pacific heart rhythm Society (APHRS), Latin America heart rhythm Society (LAHRS), and cardiac arrhythmia Society of southern Africa (CASSA). Ep Europace 21, 192-193 (2019). 
31. Aboyans, V. et al. 2017 ESC Guidelines on the Diagnosis and Treatment of Peripheral Arterial Diseases, in collaboration with the European Society for Vascular Surgery (ESVS): Document covering atherosclerotic disease of extracranial carotid and vertebral, mesenteric, renal, upper and lower extremity arteriesEndorsed by: the European Stroke Organization (ESO)The Task Force for the Diagnosis and Treatment of Peripheral Arterial Diseases of the European Society of Cardiology (ESC) and of the European Society for Vascular Surgery (ESVS). Eur. Heart J. 39, 763-816 (2018).

32. Barkat, M., Hajibandeh, S., Hajibandeh, S., Torella, F. \& Antoniou, G. A. Systematic Review and Metaanalysis of Dual Versus Single Antiplatelet Therapy in Carotid Interventions. Eur. J. Vasc. Endovasc. Surg, 53, 53-67 (2017).

33. Chan, Y. H. et al. Efficacy and Safety of Apixaban, Dabigatran, Rivaroxaban, and Warfarin in Asians With Nonvalvular Atrial Fibrillation.J. Am. Heart Assoc.7, (2018).

34. Chao, T. F. et al. Evolving Changes of the Use of Oral Anticoagulants and Outcomes in Patients With Newly Diagnosed Atrial Fibrillation in Taiwan., 138, 1485-1487 (2018).

35. Cha, M. J. et al. Effectiveness and Safety of Non-Vitamin K Antagonist Oral Anticoagulants in Asian Patients With Atrial Fibrillation., 48, 3040-3048 (2017).

\section{Figures}




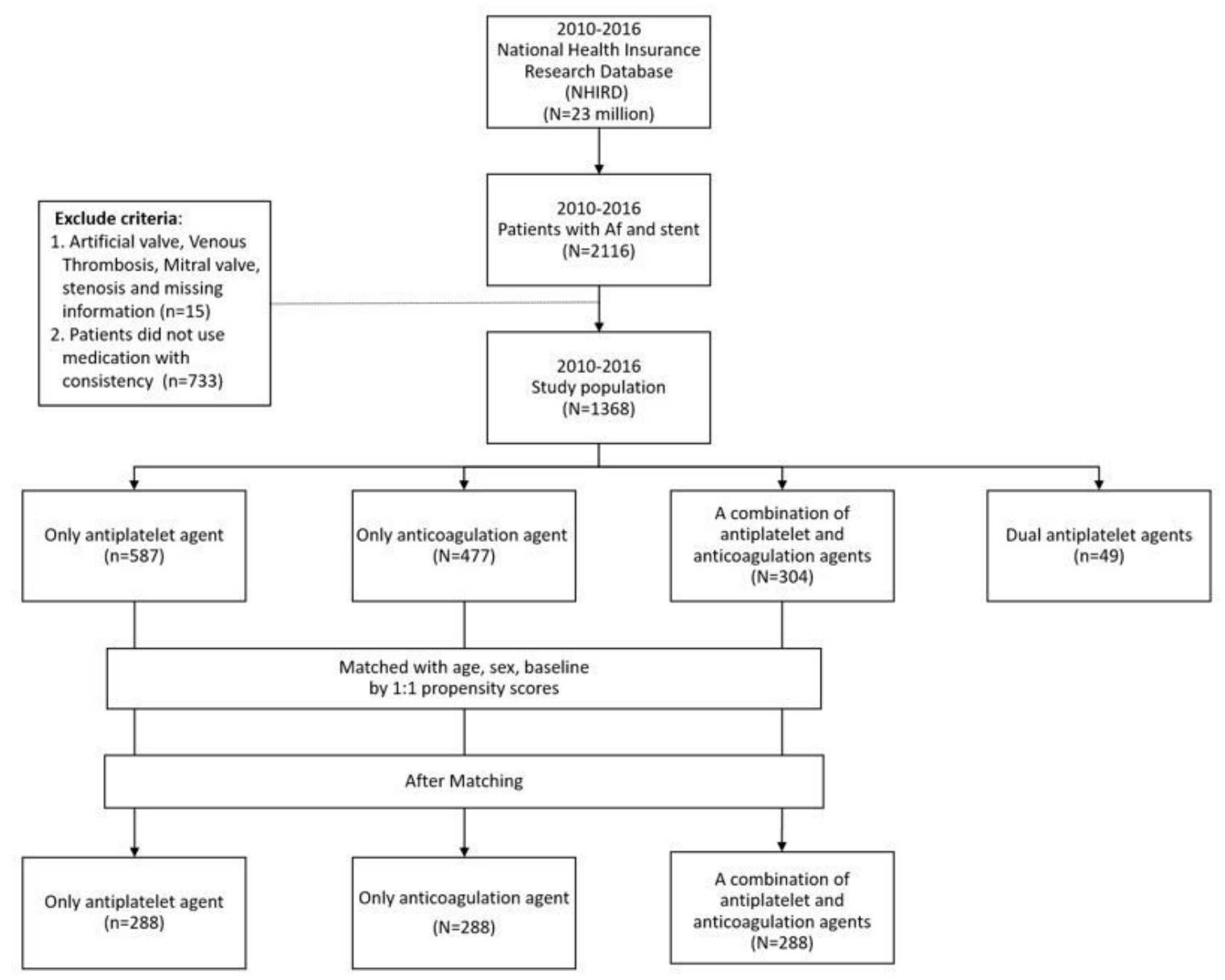

Figure 1

Study flow diagram We enrolled 2116 consecutive non-valvular atrial fibrillation patients who had successful carotid angioplasty and stent placement, with regular follow-up and consistent drug coherence, from January 1, 2010, to December 31, 2016. They were divided into groups according to treatment. We matched different therapy groups mainly according to age, sex and CHA2DS2-VASc scores for comparison of primary outcomes. 


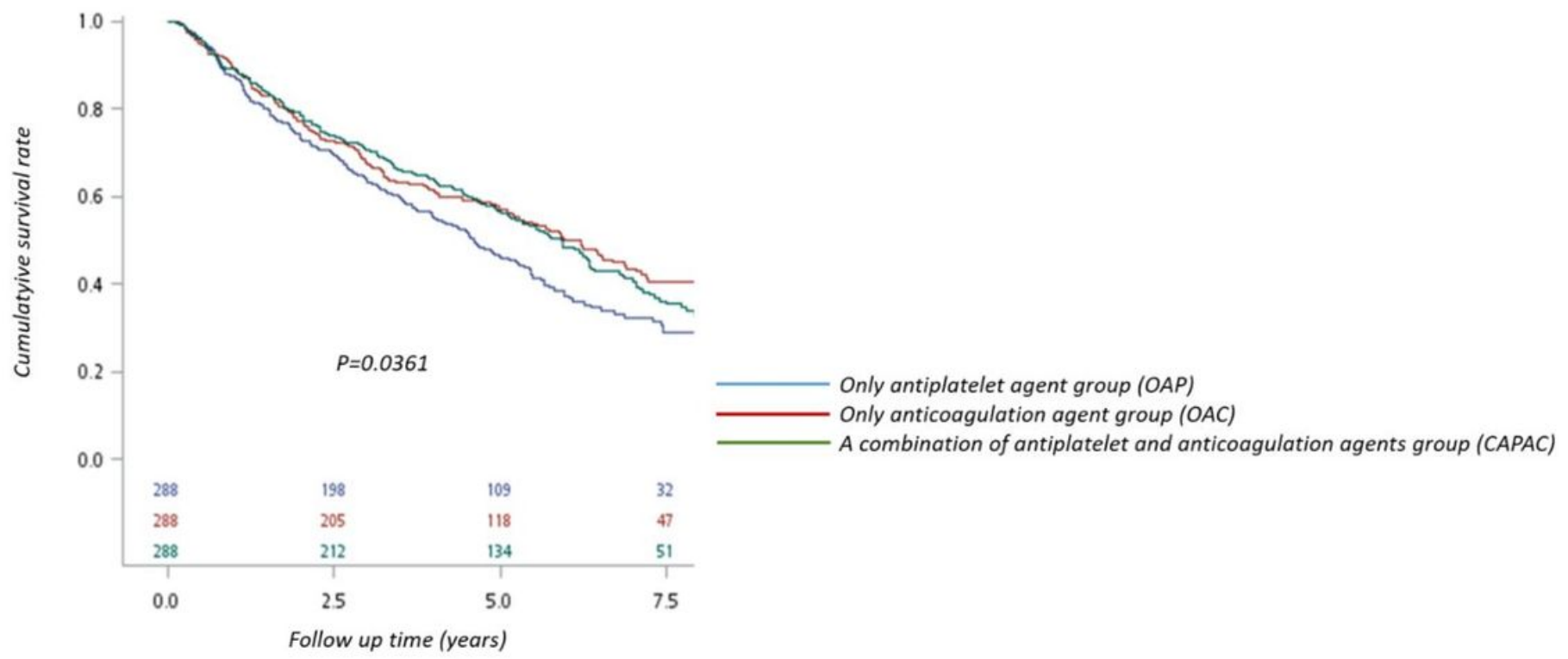

Figure 2

Kaplan-Meier analysis for cumulative survival rates, stratified by different medicine therapy groups The OAP group showed a higher mortality rate than the CAPAC and the OAC groups in the acute stage and after the follow-up 